\title{
Feasibility Analysis of EV+X Storage to Support Power System Frequency
}

\author{
Zhe Deng ${ }^{1}$, Zengping Wang ${ }^{1}$ and ${\mathrm{Ran} \mathrm{Li}^{2}}^{2}$ \\ ${ }^{1}$ North China Electric Power University, Beijing, China \\ ${ }^{2}$ University of Bath, Bath, UK
}

\begin{abstract}
With the fast growth of renewable energy generation, the power system faces the challenge of low inertia. Lower system inertia makes it more challenging to keep the frequency stable, and the conventional frequency response mechanism is not capable of ensuring frequency within the limit. In this paper, a new frequency response mechanism is proposed to help to improve the frequency performance, where electric vehicles (EV) are used as energy storage, and they will cooperate with existing primary frequency response (PFR) to form an $\mathrm{EV}+\mathrm{X}$ storage supporting power system frequency. This approach is proposed based on rigorous mathematical derivation, where the relationship between frequency and active power is quantitively analysed. To validate the new mechanism's feasibility, simulation models are built to simulate the frequency behaviour after a big disturbance, and a series of tests are conducted. Both technical and economic benefits are investigated, considering the difference in EV control strategies and the proportion of EV responses. The result shows that $\mathrm{EV}+\mathrm{X}$ storage can be a promising solution to the frequency stability problem.
\end{abstract}

\section{Introduction}

Frequency is a crucial parameter in the alternating-current (AC) power system [1]. The inertia of the power system makes it able to resist frequency fluctuation by nature. Conventional generators with heavy turbine and shaft provide sufficient inertia by nature. However, the proportion of renewable energy keeps increasing in recent years, which results in a lower level of inertia and brings a series of challenges to frequency stability [2]. To overcome the effect of low inertia, faster frequency response is in need. EV acting as energy storage is a promising solution to support power system frequency. However, the conventional frequency response source also has its advantages and can not be replaced. EV and conventional frequency response can cooperate as $\mathrm{EV}+\mathrm{X}$ storage to achieve a better frequency regulation effect.

This paper aims at investigating the effect of $\mathrm{EV}+\mathrm{X}$ storage to support power system frequency. The following chapters of this paper is organised as follows:

Section 2 analyses the relationship between active power and frequency from the perspective of mathematics. Based on this analysis, a control model is built to simulate the frequency behaviour after a disturbance. The cost of frequency response is also analysed in this section, which will be used to evaluate the economic benefit in following tests. Section 3 shows the simulation results with different control strategies and different proportions of response source. Section 4 concludes the whole work.

\section{Methodology and Modeling}

To analyse the feasibility of $\mathrm{EV}+\mathrm{X}$ storage to support power system frequency, the first step is to figure out how the frequency behaves after a disturbance. Besides the technical feasibility, the economic benefit should also be taken into consideration. The following subsections will analyse the frequency fluctuation mechanism and build a control model simulating this process, then a price model will be built based on the cost analysis.

\subsection{Mathematic Relationship between Frequency and Active Power}

In an $\mathrm{AC}$ power system, the frequency is actually the embodiment of the angular velocity of synchronous rotating components. So the analysis will start with a single synchronous generator.

For a single synchronous generator, its inertia constant is defined to be the ratio of kinetic energy stored in the rotating part of generator to power, as shown in Equation (1):

$$
H=\frac{E_{k}}{S_{n}}=\frac{\frac{1}{2} J \omega^{2}}{S_{n}}
$$

Where, $E_{k}$ is the kinetic energy stored in the turbine (J), $S_{n}$ is the nominated apparent power of the generator (VA), $J$ is the moment of inertia $\left(\mathrm{kg} \cdot \mathrm{m}^{2}\right)$.

For the rotor of the generator, the mechanical torque provided by prime mover is the torque driving it and the electromagnetic torque is the retarding torque, the

Email: dengzhe@ncepu.edu.cn 
imbalance between these two torques leads to an acceleration or deceleration, as shown in Equation (2):

$$
\frac{d \omega}{d t}=\frac{T_{m}-T_{e}}{J}
$$

Assuming the system is working at the nominated frequency, i.e. $\omega=\omega_{n}$, substituting Equation (1) into Equation (2), and replacing the torque with power $(P=$ $\omega T)$, the acceleration of rotor can be expressed as:

$$
\frac{d \omega}{d t}=\frac{T_{m}-T_{e}}{J}=\frac{\left(T_{m}-T_{e}\right) \omega_{n}^{2}}{2 H S_{n}}=\frac{\left(P_{m}-P_{e}\right) \omega_{n}}{2 H S_{n}}
$$

Where, $P_{m}$ is the mechanical power provided by prime mover, $P_{e}$ is the electromagnetic power.

Rewrite Equation (3) into the form of Equation (4):

$$
\frac{P_{m}-P_{e}}{S_{n}}=2 H \frac{d \omega}{d t} \frac{1}{\omega_{n}}=2 H \frac{2 \pi d f}{d t} \frac{1}{2 \pi f_{n}}=2 H \frac{d f}{d t} \frac{1}{f_{n}}
$$

Which can be expressed in per-unit:

$$
P_{m \text { p.u. }}-P_{\text {e p.u. }}=2 H\left(\frac{d f}{d t}\right)_{p . u .}
$$

Equation (5) is the swing equation, which gives the relationship between power imbalance and ROCOF in per-unit form. The result is based on a single generator, but can also be generalized to the whole system, where the inertia constant is defined to be the ratio of kinetic energy stored in all the spinning elements to rated apparent power, as shown in Equation (6):

$$
H_{\text {sys }}=\frac{K_{\text {Esys }}}{S_{n s y s}}=\frac{\sum H_{i} S_{i}}{S_{n s y s}}
$$

Where, $H_{\text {sys }}$ is the inertia constant of system, $K_{\text {Esys }}$ is the kinetic energy in all the rotating components among the system, $S_{n s y s}$ is the nominated apparent power of system, $H_{i}$ is the inertia constant of each machine, $S_{i}$ is the nominated apparent power of each machine [3].

\subsection{Frequency Response Model}

To focus on the most important factors, some simplification and assumptions are made in this subsection. The first assumption is that there is no system oscillation, hence all the generators can be represented by a single big generator. The second assumption is that all the synchronous generators in the system are reheat steam turbine generators since most large units participating in PFR are this type.

As introduced before, PFR is conducted by synchronous generators with the governor. Taking reheat steam turbine generators as the example, ignoring other factors, the behaviour of reheat steam turbine generators is predominated by the time constant of reheater time constant [4]. Assuming the proportion of power coming from high-pressure turbine is $K_{H P}$, the proportion of power coming from low-pressure turbine will be $1-K_{H P}$. The synchronous generator response model can be represented by Figure 1, where $T_{R}$ is the time constant of reheater.

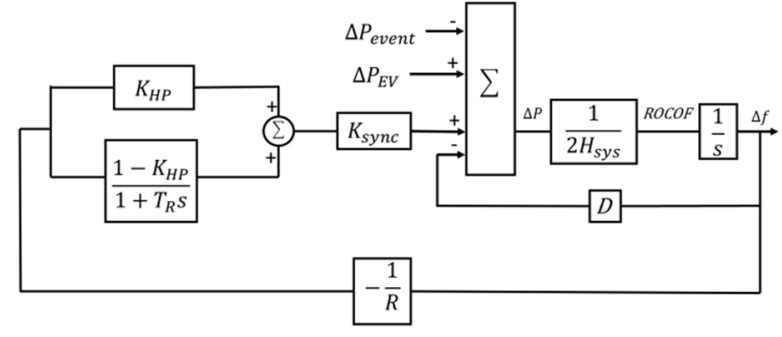

Figure 1. Control model of frequency response

\subsection{EV Models}

The general idea about EV supporting frequency is to make it output power when the frequency contingency occurs, but the details can be complicated. Different control strategies also have an influence on the result of frequency response. According to EV's control strategy of releasing power, two response modes are proposed:

(1) Time-linear Output, which is the simplest model, where the output power of EV is proportional to time once the response is triggered, until reaching the maximum output power. The relationship between power and time is expressed by Equation (7).

$$
=\left\{\begin{array}{lll}
P_{\text {linear }} t, & \text { if } & k_{\text {linear }} t \leq P_{\text {max }} \\
P_{\text {max }}, & \text { if } & k_{\text {linear }} t>P_{\text {max }}
\end{array}\right.
$$

(2) Droop Control, which emulates the droop characteristic of the generator. In this mode, the output power of EV is linear over frequency deviation, as shown in Equation (8).

$$
\begin{aligned}
& P_{\text {droop }} \\
& =\left\{\begin{array}{lll}
k_{\text {droop }} \Delta f, & \text { if } & k_{\text {droop }} \Delta f \leq P_{\max } \\
P_{\text {max }}, & \text { if } & k_{\text {droop }} \Delta f>P_{\max }
\end{array}\right.
\end{aligned}
$$

\subsection{Cost Analysis and Price Model}

For conventional PFR, the cost includes two parts, the holding cost and energy cost. To compare the cost of conventional PFR and EV response, it is assumed that they have the same holding cost and energy cost. However, the battery degradation must be taken into consideration. EV participating in frequency response will charge and discharge more frequently, thus will have a shorter battery life. To compensate for the additional cost of battery replacement, the price of $\mathrm{EV}$ response will be higher than conventional PFR. Assuming the degree of degradation is positively correlated with the amount of charge and discharge. If the cost of battery replacement is normalized to every kWh throughput, the battery degradation cost can be expressed as Equation (9).

$$
C_{d e g}=\frac{1000 C_{r e p}}{n_{c y c} \times C a p}
$$

Where, $C_{d e g}$ is the battery degradation cost normalized to every $\mathrm{kWh}$ throughput energy, in pounds/MWh, $C_{r e p}$ is the battery replacement cost, $n_{c y c}$ is the number of cycles when the battery needs to be replaced, Cap is the capacity of battery, in kWh.

As both energy cost and battery degradation cost are proportional to energy consumed, they will be integrated 
together as equivalent energy price $C_{\text {energy_eq }}$, as shown in Equation (10).

$$
\begin{aligned}
& C_{\text {energy_eq }} \\
& =\left\{\begin{array}{l}
C_{\text {energy }} \text { for conventional PFR } \\
C_{\text {energy }}+C_{\text {deg }} \text { for EV response }
\end{array}\right.
\end{aligned}
$$

To compare the cost performance in different scenarios, the energy consumption of frequency response in 30 s after the generation loss is recorded and the total cost is calculated as Equation (11).

$$
\text { Cost }=C_{\text {energy }} \times E_{P F R}+\left(C_{\text {energy }}+C_{\text {deg }}\right) \times E_{E V}
$$

\section{Simulation and Result Analysis}

Based on the analysis above, a control model is built in MATLAB/SIMULINK to simulate the frequency behaviour after a significant disturbance. A series of test scenarios are designed to investigate the performance of different response mode, as well as EV proportion.

The test scenarios are designed delicately based on a real event. According to the Technical Report on the events of 9 August 2019 [5], the load is 29GW when the event happened and there is $32 \mathrm{GW}$ available, among which $50 \%$ is provided by conventional synchronous generators. Assuming the power factor is 0.85 , which is typical for British Power system [6], the base value is set to be $29 \div 0.85=37.647 \mathrm{GVA}$. Considering the potential loss of the maximum infeed unit, Sizewell nuclear station with the capacity of $1260 \mathrm{MW}$, the minimum reserve should be $1260 \mathrm{MW}$, i.e. 0.03347 in per-unit. In the test, a $1260 \mathrm{MW}$ generation loss is simulated and the total reserve capacity is $1260 \mathrm{MW}$, which is provided by EV and PFR together. Other parameters are listed in Table 1.

Table 1. Parameters of the testing system

\begin{tabular}{ccccc}
\hline$K_{H P}$ & $K_{S G}$ & $H$ & $D$ & $R$ \\
\hline $10 \%$ & $50 \%$ & $3 \mathrm{~s}$ & 1 & 0.05 \\
\hline
\end{tabular}

The average price of the day-ahead market in the past ten years (from [7]) is taken as the energy cost, which is 45.7 pounds/MWh. As for battery degradation, $C_{\text {deg }}$ is set to 211 pounds/MWh $[8,9,10]$.

Based on this analysis, the equivalent energy for conventional PFR and EV response are listed below:

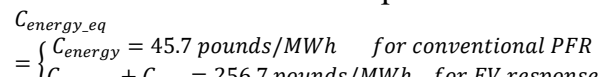

\subsection{Test Result of Time-linear Output}

In this section, the influence of EV response parameters such as ramp-up rate $\left(k_{\text {linear }}\right)$ and response time delay $\left(t_{d}\right)$ are investigated first. Then the proportion of EV response is adjusted to find an optimal value.

\subsubsection{Influence of $\boldsymbol{k}_{\text {linear }}$}

Here the percentage of EV response is set to $50 \%$, and the time delay is set to 0.5 seconds. The ramp-up rate $k_{\text {linear }}$ ranges from $200 \mathrm{MW} / \mathrm{s}$ to $1000 \mathrm{MW} / \mathrm{s}$, with a step length of $200 \mathrm{MW} / \mathrm{s}$. The frequency performance, power consumption and cost are shown in Figure 2.

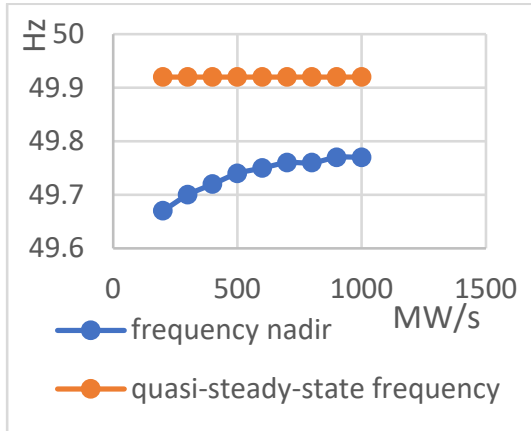

(a) frequency

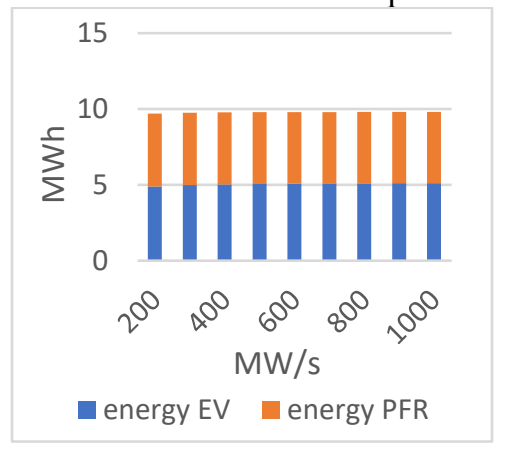

(b) power consumption

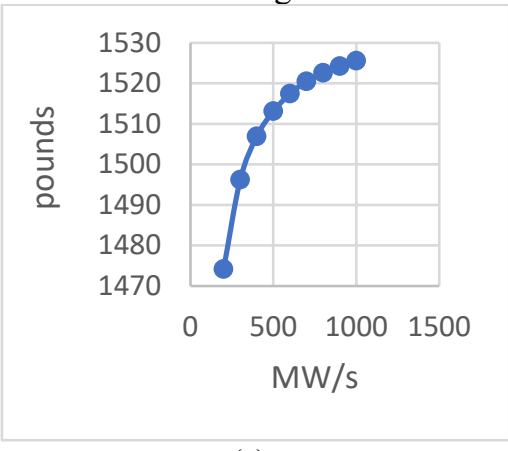

(c) $\operatorname{cost}$

Figure 2. Performance of different $k_{\text {linear }}$

The result indicates that the frequency nadir increases with $k_{\text {linear }}$, but when $k_{\text {linear }}$ is larger, the increase tends to be slower. The quasi-steady-state frequency does not change with $k_{\text {linear }}$. The energy provided by EV increase slightly with the increase of $k_{\text {linear }}$. As the energy from EV only increases a little, the cost does not increase significantly.

\subsubsection{Influence of $\boldsymbol{t}_{\boldsymbol{d}}$}

To investigate how time delay $t_{d}$ affects the frequency behaviour, another set of cases are designed, where $k_{\text {linear }}$ is kept $700 \mathrm{MW} / \mathrm{s}$ and the proportion of EV stays at $50 \%$. The time delay is set to $0.2 \mathrm{~s}$ at the first case and increases by $0.2 \mathrm{~s}$ until $1.8 \mathrm{~s}$. The performance is shown in Figure 3. 


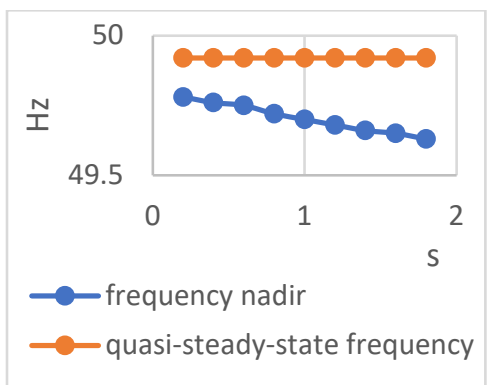

(a) frequency

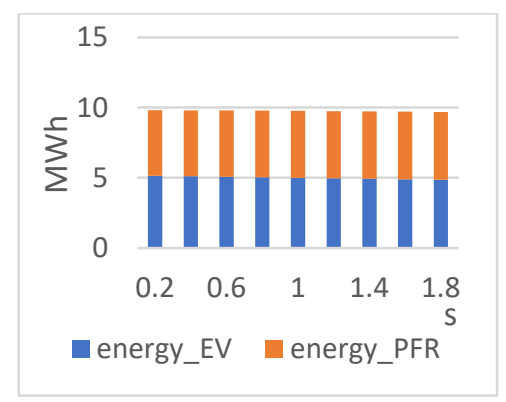

(b) power consumption

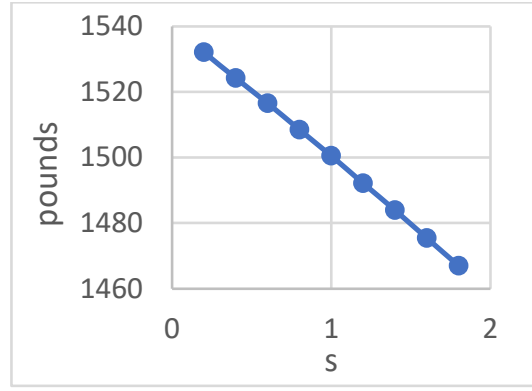

(c) $\operatorname{cost}$

Figure 3. Performance of different $t_{d}$

The curve shows that the frequency nadir decreases as $t_{d}$ increase and the relationship is nearly linear, which indicates that response speed is crucial to frequency stability. The quasi-steady-state frequency stays the same as the total response capacity is the same. As the time delay increases, the energy coming from EV decreases, while the energy provided by PFR increases. As a result, the total energy consumption ranges from $9.808 \mathrm{MWh}$ to 9.677MWh, which do not change with $t_{d}$ obviously. This indicates that time delay does not affect power consumption significantly. (a) frequency

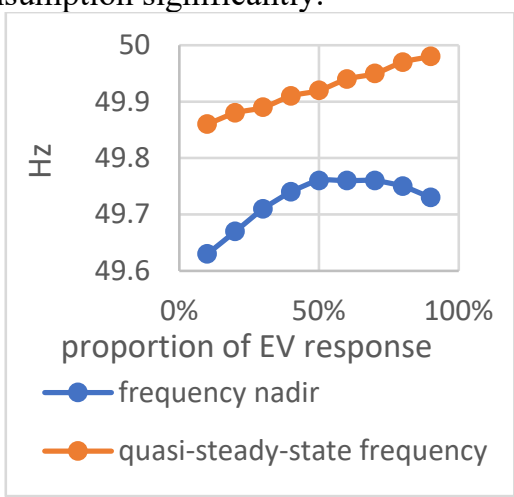

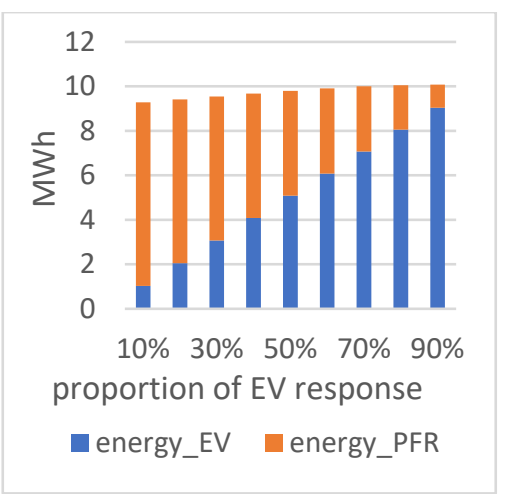

(b) power consumption

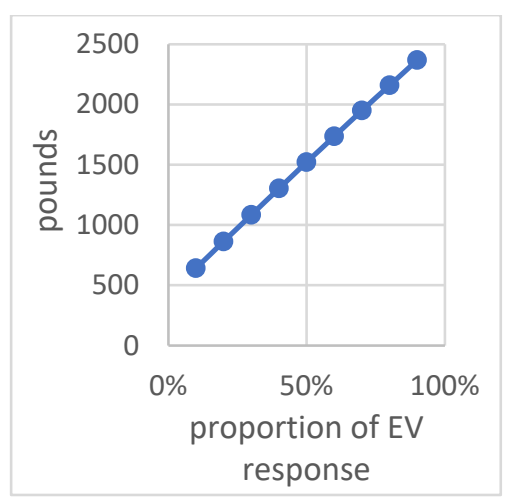

(c) $\operatorname{cost}$
Figure 4. Performance of different EV response proportion
As the EV response increase, though the total response capacity is the same, the quasi-steady-state frequency increase from $49.86 \mathrm{~Hz}$ to $49.98 \mathrm{~Hz}$. However, the frequency nadir shows a different trend. The energy from EV increases with EV's proportion and the total energy consumption tends to increase. Because the equivalent energy price of EV response is much more expensive than conventional PFR, the total cost is nearly linear to the proportion of EV. This result implies that even if the timelinear EV response is able to improve the frequency behaviour, it may not be cost-efficient enough.

\subsection{Test Result of Droop Control}

Droop control simulates the behaviour of governors. The following paragraphs will show the test result.

\subsubsection{Influence of EV Proportion in Time-linear}

To find an optimal proportion of EV response, test cases are designed to have the same EV parameters $\left(k_{\text {linear }}=700 \mathrm{MW} / \mathrm{s}, t_{d}=0.5 \mathrm{~s}\right)$, but the percentage of EV response changes from $10 \%$ to $90 \%$, while the total reserve is kept the same. Figure 4 shows the performance.

\subsubsection{Influence of $\boldsymbol{k}_{\text {droop }}$}

To analyse the influence of $k_{\text {droop }}$ on frequency response result, the proportion of $\mathrm{EV}$ is set to $50 \%$, while the other $50 \%$ is provided by conventional PFR. In this circumstance, the EV capacity is 0.0167 in per-unit. If the $\mathrm{EV}$ gives a full delivery when the frequency falls to the statutory limit $\left( \pm 0.5 \mathrm{~Hz}\right.$, i.e. 0.01 in per-unit), the $k_{\text {droop }}$ will be 1.67 . To achieve better response effect, $k_{\text {droop }}$ can be set to a larger value. So in the tests $k_{\text {droop }}$ ranges from 1.67 to 10 . The test result is shown in Figure 5. 


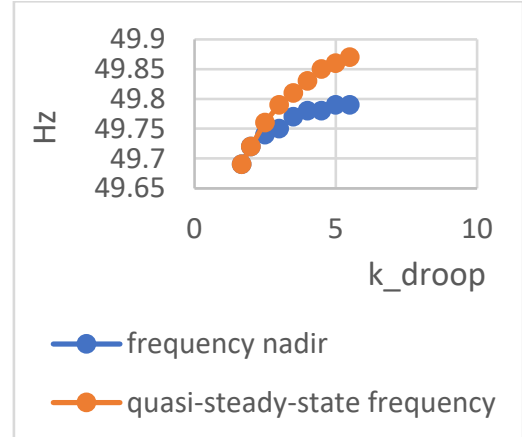

(a) frequency

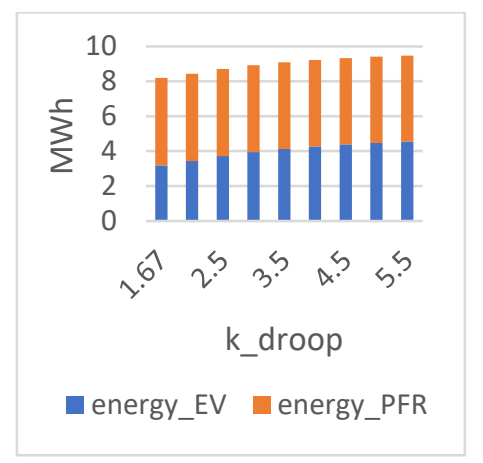

(b) power consumption

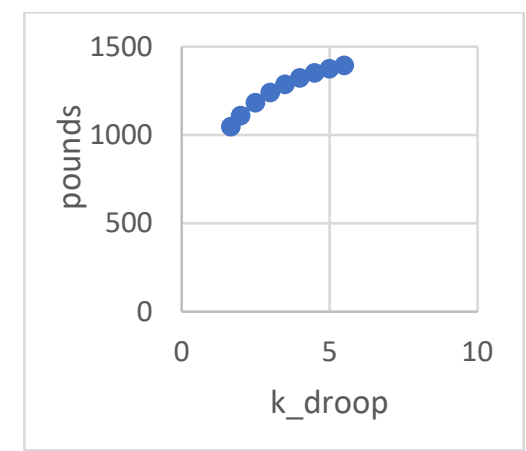

(c) $\operatorname{cost}$

Figure 5. Performance with different $k_{\text {droop }}$

The increase of frequency is faster when $k_{\text {droop }}$ is smaller. The quasi-steady-state frequency keeps increasing with $k_{\text {droop }}$. Here the energy provided by PFR does not change significantly, but the energy from EV increases as $k_{\text {droop }}$ increases. As a result, the total energy consumption increases from 8.200MWh to $9.477 \mathrm{MWh}$ as $k_{\text {droop }}$ changes from 1.67 to 5.5 . Larger $k_{\text {droop }}$ means more power injected at the beginning of a frequency event, which leads to the difference on total energy consumption. The increase of $k_{\text {droop }}$ brings an increase to total cost. The energy cost is 1046.664 pounds when $k_{\text {droop }}$ is 1.67 , and then increases to 1393.363 pounds when $k_{\text {droop }}$ is 5.5 , which increases by $33.12 \%$.

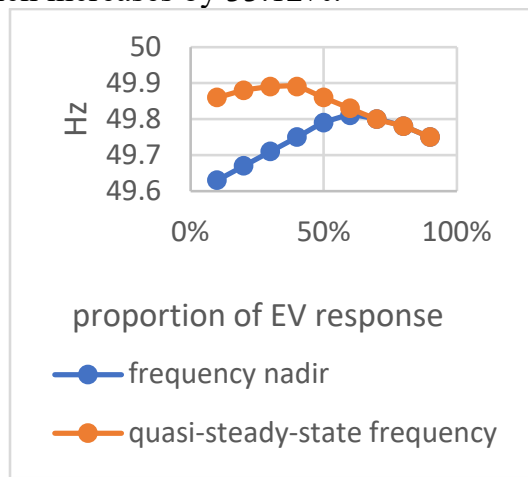

(a) frequency

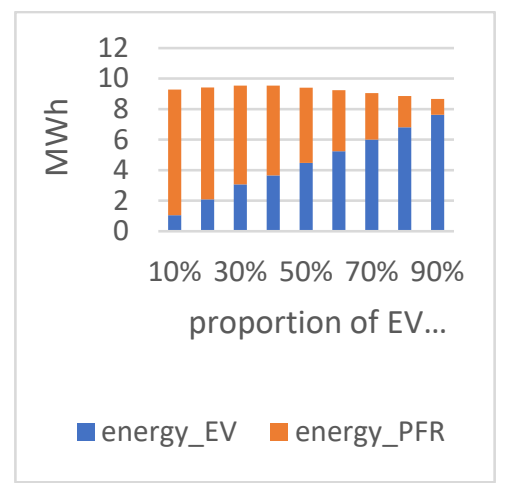

(b) power consumption

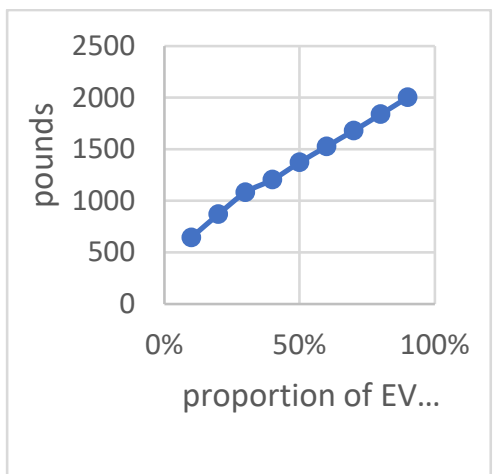

(c) $\cos t$

Figure 6. Performance of different EV response proportion

Figure 6 shows the frequency performance of different EV response proportion. The curve indicates that both frequency nadir and quasi-steady-state frequency increase first, but then goes down. The energy contributed by EV increase with EV proportion. The total energy consumption shows the same trend as quasi-steady-state frequency. When it comes to the cost, the increase of EV response will result in a significant increase in cost. In the testing cases, the total cost increases from 645.2377 pounds to 2003.919 pounds, which is an increase up to 3 times.

\section{Conclusion}

Facing the low inertia problem, this paper analyses the feasibility of using $\mathrm{EV}+\mathrm{X}$ storage to support power system frequency. A frequency response model in MATLAB/SIMULINK is built to simulate the frequency behaviour after a disturbance. The cost difference between EV response and conventional PFR is also considered. Different control methods, including time-linear output and droop control are implemented. For each method, the influence of control parameter is investigated. The test results show that conventional PFR has its advantages and should not be totally replaced by EV response. EV acting as energy storage can be a good supplement to the existing frequency response mechanism. The optimal proportion depends on the control method and specific parameter tuning. EV acting as energy storage could be a promising way to solve the low inertia problem in the power system. This paper analyses the feasibility in theory, but more research can be done in the future. 


\section{Reference}

1. Kundur, P., Paserba, J., Ajjarapu, V., Andersson, G., Bose, A., Canizares, C., Hatziargyriou, N., Hill, D., Stankovic, A., Taylor, C. and Van Cutsem, T., 2004. Definition and classification of power system stability IEEE/CIGRE joint task force on stability terms and definitions. IEEE transactions on Power Systems, 19(3), pp.1387-1401.

2. Tielens, P. and Van Hertem, D., 2016. The relevance of inertia in power systems. Renewable and Sustainable Energy Reviews, 55, pp.999-1009.

3. Weedy, B.M., Cory, B.J., Jenkins, N., Ekanayake, J.B. and Strbac, G., 2012. Electric power systems. John Wiley \& Sons.

4. Anderson, P.M. and Mirheydar, M., 1990. A loworder system frequency response model. IEEE Transactions on Power Systems, 5(3), pp.720-729.

5. National Grid ESO, 2019. Technical Report on the events of 9 August 2019 [Online]. Available from: https://www.ofgem.gov.uk/system/files/docs/2019/0 9/eso_technical_report_-_final.pdf. [Accessed 28 August 2020].

6. Hong, Q., Nedd, M., Norris, S., Abdulhadi, I., Karimi, M., Terzija, V., Marshall, B., Bell, K. and Booth, C., 2019. Fast frequency response for effective frequency control in power systems with low inertia. The Journal of Engineering, 2019(16), pp.1696-1702.

7. Ofgem, 2020. Electricity prices: Day-ahead baseload contracts-monthly average (GB) [Online]. Available from: https://www.ofgem.gov.uk/dataportal/electricity-prices-day-ahead-baseload-

contracts-monthly-average-gb. [Accessed 2 September 2020].

8. Green Car Reports, 2014. Nissan Leaf New Battery Cost: $\$ 5500$ for Replacement with Heat-resistant Chemistry [Online]. Available from: https://www.greencarreports.com/news/1092983_nis san-leaf-battery-cost-5500-for-replacement-withheat-resistant-chemistry [Accessed 2 September 2020].

9. Green Car Reports, 2017. How much is a replacement Chevy Bolt EV electric-car battery? [Online]. Available from: https://www.greencarreports.com/news/1110881_ho w-much-is-a-replacement-chevy-bolt-ev-electriccar-battery [Accessed 2 September 2020].

10. Xu, B., Oudalov, A., Ulbig, A., Andersson, G. and Kirschen, D.S., 2016. Modeling of lithium-ion battery degradation for cell life assessment. IEEE Transactions on Smart Grid, 9(2), pp.1131-1140. 